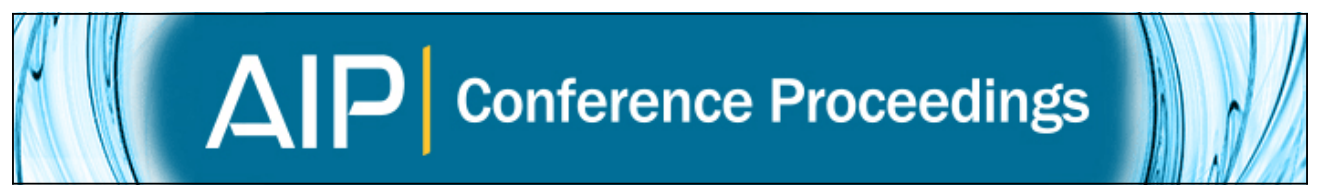

\title{
RESOLUTIONS IN COTORSION THEORIES
}

Karen Akinci and Rafail Alizade

Citation: AIP Conference Proceedings 1309, 761 (2010); doi: 10.1063/1.3525203

View online: http://dx.doi.org/10.1063/1.3525203

View Table of Contents:

http://scitation.aip.org/content/aip/proceeding/aipcp/1309?ver=pdfcov

Published by the AIP Publishing

\section{Articles you may be interested in}

An Application of Variational Theory to an Integrated Walrasian Model of Exchange, Consumption and Production

AIP Conf. Proc. 1281, 269 (2010); 10.1063/1.3498447

Symposium on Anisotropic Mesh Generation: Theory and Practical Aspects

AIP Conf. Proc. 1281, 1554 (2010); 10.1063/1.3498098

The Importance of the Numerical Resolution of the Laplace Equation in the optimization of a Neuronal Stimulation Technique

AIP Conf. Proc. 1281, 1199 (2010); 10.1063/1.3497884

Numerical generation of hyperspherical harmonics for tetra-atomic systems

J. Chem. Phys. 125, 133505 (2006); 10.1063/1.2218515

Spaceship with a thruster-one body, one force

Am. J. Phys. 73, 500 (2005); 10.1119/1.1858451 


\title{
RESOLUTIONS IN COTORSION THEORIES
}

\author{
KAREN AKINCI AND RAFAIL ALIZADE
}

\begin{abstract}
We consider the $\lambda_{-}(\mu-)$ and $\bar{\lambda}-\left(\bar{\mu}^{-}\right)$dimensions of modules taken under a cotorsion theory $(\mathcal{F}, \mathcal{C})$ satisfying the Hereditary Condition, and establish some inequalities between the dimensions of the modules of a short exact sequence, not necessarily $\operatorname{Hom}(\mathcal{F},-)$ exact. We investigate the question of whether the property of having a (special) $\mathcal{F}$ - or $\mathcal{C}$-resolution of length $n$ is resolving, closed under extensions or coresolving and establish some inequalities connecting the $\lambda_{-}\left(\mu_{-}\right)$and $\bar{\lambda}_{-}\left(\bar{\mu}^{-}\right)$dimensions of modules in a short exact sequence.
\end{abstract}

\section{INTRODUCTION}

Throughout a module will mean a unitary left $R$-module over an arbitrary but fixed ring $R$ with identity.

A cotorsion theory (see [8] ) is a pair of classes of modules $(\mathcal{F}, \mathcal{C})$ such that

$$
\mathcal{F}={ }^{\perp} \mathcal{C}=\left\{F \mid \operatorname{Ext}^{1}(F, C)=0 \text { for all } C \in \mathcal{C}\right\}
$$

and

$$
\mathcal{C}=\mathcal{F}^{\perp}=\left\{C \mid \operatorname{Ext}^{1}(F, C)=0 \text { for all } F \in \mathcal{F}\right\} .
$$

A partial left $\mathcal{F}$-resolution (or partial $\mathcal{F}$-projective resolution) of a module $M$ of length $n$ is a complex $F_{n} \stackrel{d_{n}}{\rightarrow} F_{n-1} \rightarrow \ldots \rightarrow F_{1} \stackrel{d_{1}}{\rightarrow} F_{0} \stackrel{d_{0}}{\rightarrow} M \rightarrow 0$ with each $F_{i} \in \mathcal{F}$, which is $\operatorname{Hom}(F,-)$ exact for every $F \in \mathcal{F}$. Similarly a partial right $\mathcal{C}$-resolution of a module $M$ of length $n$ is a complex $0 \rightarrow M \stackrel{e_{0}}{\rightarrow} C_{0} \stackrel{e_{1}}{\rightarrow} C_{1} \rightarrow \ldots \rightarrow C_{n-1} \stackrel{e_{n}}{\rightarrow} C_{n}$ with each $C_{i} \in \mathcal{C}$, which is $\operatorname{Hom}(-, C)$ exact for every $C \in \mathcal{C}$. Taken under a cotorsion theory $(\mathcal{F}, \mathcal{C})$, an $\mathcal{F}$-resolution is normally left and a $\mathcal{C}$-resolution is normally right, this will not be stated where there is no danger of ambiguity. If $\operatorname{Ker} d_{i} \in \mathcal{C}$ for all $i$, then the partial $\mathcal{F}$-resolution is called special and similarly the partial $\mathcal{C}$-resolution above is special if Coker $e_{i} \in \mathcal{F}$ for all $i$.

Definition 1.1. The $\lambda$-dimension $(\bar{\lambda}$-dimension) of $M$ is defined as follows: $\lambda(M)=$ $n(\bar{\lambda}(M)=n)$ if there is a partial $\mathcal{F}$-resolution (special partial $\mathcal{F}$-resolution) $F_{n} \stackrel{d_{n}}{\longrightarrow}$ $F_{n-1} \longrightarrow \ldots \longrightarrow F_{1} \stackrel{d_{1}}{\longrightarrow} F_{0} \stackrel{d_{0}}{\longrightarrow} M \longrightarrow 0$ of $M$ of length $n$ and if there is no longer such complex. If there is no partial $\mathcal{F}$-resolution (special partial $\mathcal{F}$-resolution) then we say that $\lambda(M)=-1(\bar{\lambda}(M)=-1)$, and if there exists a partial $\mathcal{F}$-resolution (special partial $\mathcal{F}$-resolution) for every $n \geq 0$ we say that $\lambda(M)=\infty(\bar{\lambda}(M)=\infty)$. The partial (special

Key words and phrases. Cotorsion Theory, Hereditary Condition, Special left $\mathcal{F}$-resolution, Special right $\mathcal{C}$-resolution, $\lambda$ -,$\mu-, \bar{\lambda}$-, $\bar{\mu}$ - dimensions. 
partial) $\mathcal{C}$-resolution and $\mu$-dimension ( $\bar{\mu}$-dimension) for a class of $\mathcal{C}$ modules are defined dually.

For every $F \in \mathcal{F}$ we have special $\mathcal{F}$-resolution $\ldots \rightarrow 0 \rightarrow \ldots \rightarrow 0 \rightarrow F \rightarrow F \rightarrow 0$, so $\lambda(F)=\bar{\lambda}(F)=\infty$. Similarly $\mu(C)=\bar{\mu}(C)=\infty$ for every $C \in \mathcal{C}$.

We study the notions of $\bar{\lambda}$-dimension (and $\bar{\mu}$-dimension) when $(\mathcal{F}, \mathcal{C})$ is a cotorsion theory satisfying the Hereditary Condition $(H C)$ (see [3]), that is, $\operatorname{Ext}^{2}(F, C)=0$ for every $F \in \mathcal{F}$ and $C \in \mathcal{C}$, or equivalently, $\mathcal{F}$ is resolving, or $\mathcal{C}$ is coresolving. Recall that a class $\mathcal{A}$ of modules containing all projective (injective) modules is called resolving (coresolving) if for every short exact sequence of modules $0 \rightarrow A \rightarrow B \rightarrow C \rightarrow 0$ the condition $B, C \in \mathcal{A}$ $(A, B \in \mathcal{A})$ implies $A \in \mathcal{A}(C \in \mathcal{A})$.

The following example of a cotorsion theory not satisfying $H C$ is given in the proof of Proposition 3.6 in [4]. Recall that a module $C$ is called weakly cotorsion if it is cotorsion in the Matlis sense, that is, $\operatorname{Ext}^{1}(Q, C)=0$ (where $Q$ is the field of fractions of $R$ ) (see $[7]$ ), and a module $F$ is strongly flat if $\operatorname{Ext}^{1}(F, C)=0$ for all weakly cotorsion modules $C$. Let $R$ be a valuation domain which is not a Matlis domain, i.e. pr. $\operatorname{dim} Q>1$ and let $0 \rightarrow H \rightarrow F \rightarrow Q \rightarrow 0$ be a free presentation of $Q$. Then $F$ and $Q$ are strongly flat but $H$ is not (see the proof of Prop. 3.6 in [4]). So the cotorsion theory $(\mathcal{S} F, \mathcal{W} C)$, where $\mathcal{S} F$ is the class of strongly flat modules and $\mathcal{W} C$ is the class of the weakly cotorsion modules, does not satisfy $H C$.

\section{2. $\bar{\lambda}$ - AND $\bar{\mu}$-DIMENSIONS}

The following theorem is similar to Theorem 8.6.14 of [5] where the exact sequence $0 \rightarrow M^{\prime} \rightarrow M \rightarrow M^{\prime \prime} \rightarrow 0$ is $\operatorname{Hom}(\mathcal{F},-)$ exact. We can remove this condition and prove the stronger case for any cotorsion theory $(\mathcal{F}, \mathcal{C})$ that satisfies $H C$.

Theorem 2.1. If the cotorsion theory $(\mathcal{F}, \mathcal{C})$ satisfies $H C$ and $0 \rightarrow M^{\prime} \rightarrow M \rightarrow M^{\prime \prime} \rightarrow 0$ is exact then $\bar{\lambda}(M) \geq \min \left(\bar{\lambda}\left(M^{\prime}\right), \bar{\lambda}\left(M^{\prime \prime}\right)\right)$.

Proof. Let $\min \left(\bar{\lambda}\left(M^{\prime}\right), \bar{\lambda}\left(M^{\prime \prime}\right)\right)=n$, by induction on $n$ we will prove that $\bar{\lambda}(M) \geq n$. For $n=-1$ there is nothing to prove. If $n=0$, then both $M^{\prime}$ and $M^{\prime \prime}$ have special $\mathcal{F}$-precovers. By Theorem 3.1 of [1], $M$ also has a special $\mathcal{F}$-precover, so $\bar{\lambda}(M) \geq 0$. Assume that for all $n \leq k$ the inequality holds and let $n=k+1$. Given $\bar{\lambda}\left(M^{\prime}\right), \bar{\lambda}\left(M^{\prime \prime}\right) \geq k+1$, then there are special $\mathcal{F}$-resolutions: $F_{k}^{\prime} \stackrel{d_{k}^{\prime}}{\longrightarrow} F_{k-1}^{\prime} \longrightarrow \ldots \longrightarrow F_{1}^{\prime} \stackrel{d_{1}^{\prime}}{\longrightarrow} F_{0}^{\prime} \stackrel{f}{\longrightarrow} M^{\prime} \longrightarrow 0$ and $F_{k}^{\prime \prime} \stackrel{d_{k}^{\prime \prime}}{\longrightarrow} F_{k-1}^{\prime \prime} \longrightarrow \ldots \longrightarrow F_{1}^{\prime \prime} \stackrel{d_{1}^{\prime \prime}}{\longrightarrow} F_{0}^{\prime \prime} \stackrel{g}{\longrightarrow} M^{\prime \prime} \longrightarrow 0$. By the proof of Theorem 3.1 in [1] we have the following diagram with exact rows

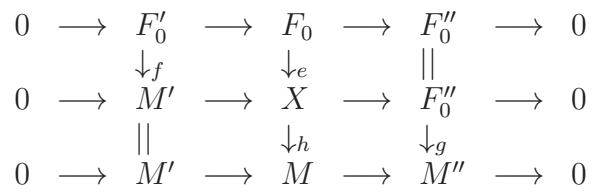


From this diagram we obtain the following commutative diagram whose columns and rows are exact by the $3 \times 3$ Lemma.

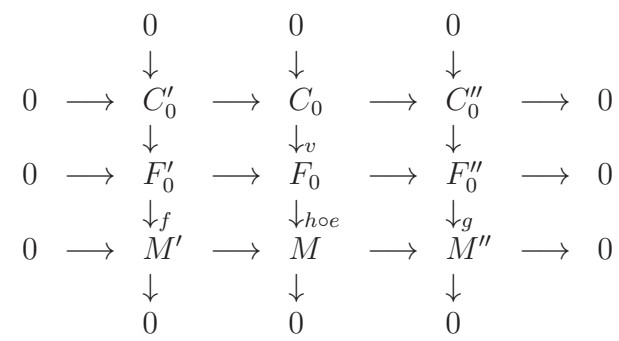

where $C_{0}^{\prime}=\operatorname{Ker} f \in \mathcal{C}$ and $C_{0}^{\prime \prime}=\operatorname{Ker} g \in \mathcal{C}$. This means $C_{0}=\operatorname{Ker}(h \circ e) \in \mathcal{C}$ also. Since $F_{0}^{\prime}, F_{0}^{\prime \prime} \in \mathcal{F}$ we have that $F_{0} \in \mathcal{F}$ also. For $C_{0}^{\prime}$ and $C_{0}^{\prime \prime}$ there are special $\mathcal{F}$-resolutions: $F_{k}^{\prime} \stackrel{d_{k}^{\prime}}{\longrightarrow} F_{k-1}^{\prime} \longrightarrow \ldots \longrightarrow F_{1}^{\prime} \longrightarrow C_{0}^{\prime} \longrightarrow 0$ and $F_{k}^{\prime \prime} \stackrel{d_{k}^{\prime \prime}}{\longrightarrow} F_{k-1}^{\prime \prime} \longrightarrow \ldots \longrightarrow F_{1}^{\prime \prime} \longrightarrow C_{0}^{\prime \prime} \longrightarrow 0$, so $\bar{\lambda}\left(C_{0}^{\prime}\right) \geq k$ and $\bar{\lambda}\left(C_{0}^{\prime \prime}\right) \geq k$, and by the inductive assumption $\bar{\lambda}\left(C_{0}\right) \geq k$. That is, $C_{0}$ has a special $\mathcal{F}$-resolution $F_{k} \longrightarrow F_{k-1} \longrightarrow \ldots \longrightarrow F_{1} \stackrel{u}{\longrightarrow} C_{0} \longrightarrow 0$. Then $F_{k} \longrightarrow F_{k-1} \longrightarrow \ldots \longrightarrow F_{1} \stackrel{v \circ u}{\longrightarrow} F_{0} \longrightarrow M \longrightarrow 0$, where $v: C_{0} \rightarrow F_{0}$ is the inclusion map, gives a special $\mathcal{F}$-resolution of $M$, that is $\bar{\lambda}(M) \geq k+1$ as required.

The following theorem shows that if $\bar{\lambda}(M)=n>k \geq 0$ then every special $\mathcal{F}$-resolution of length $k$ can be extended to a special $\mathcal{F}$-resolution of length $n$. Meaning that the $\bar{\lambda}$-dimension of a module $M$ does not depend on the choice of the special $\mathcal{F}$-resolution. The analogous result for $\mathcal{F}$-resolutions was proved in [5] (Prop 8.6.6).

Theorem 2.2. If the cotorsion theory $(\mathcal{F}, \mathcal{C})$ satisfies $H C, \bar{\lambda}(M) \geq n>k \geq 0$ and $F_{k} \stackrel{d_{k}}{\longrightarrow} F_{k-1} \longrightarrow \ldots \longrightarrow F_{1} \stackrel{d_{1}}{\longrightarrow} F_{0} \stackrel{d_{0}}{\longrightarrow} M \longrightarrow 0$ is a partial special left $\mathcal{F}$-resolution of length $k$ of $M$, then $\bar{\lambda}\left(L_{k}\right) \geq n-k-1$ where $L_{k}=\operatorname{Ker} d_{k} \in \mathcal{C}$. In particular, if $\bar{\lambda}(M)=n$, then $\bar{\lambda}\left(L_{k}\right)=n-k-1$.

Proof. This theorem is again proven by induction on $k$. For $k=0$, applying Theorem 8.6.16 of [5], to the exact sequence $0 \rightarrow L_{0} \rightarrow F_{0} \rightarrow M \rightarrow 0$, we see that $\bar{\lambda}\left(L_{0}\right) \geq$ $\min \left(\bar{\lambda}\left(F_{0}\right), \bar{\lambda}(M)-1\right)=\bar{\lambda}(M)-1 \geq n-0-1$. Assume that $\bar{\lambda}\left(L_{k}\right) \geq n-k-1$. Applying Theorem 8.6.16. of [5] to the exact sequence $0 \rightarrow L_{k+1} \rightarrow F_{k+1} \rightarrow L_{k} \rightarrow 0$, we get $\bar{\lambda}\left(L_{k+1}\right) \geq \min \left(\bar{\lambda}\left(F_{k+1}\right), \bar{\lambda}\left(L_{k}\right)-1\right)=\bar{\lambda}\left(L_{k}\right)-1 \geq n-k-1-1=n-(k+1)-1$.

Now suppose that $\bar{\lambda}(M)=n, \bar{\lambda}\left(L_{k}\right)=s$ and let $G_{s} \rightarrow G_{s-1} \rightarrow \ldots \rightarrow G_{1} \rightarrow L_{k} \rightarrow 0$ be a special $\mathcal{F}$-resolution of $L_{k}$. Then $G_{s} \rightarrow \ldots \rightarrow G_{1} \rightarrow F_{k} \rightarrow \ldots \rightarrow F_{0} \rightarrow M \rightarrow 0$ is a special $\mathcal{F}$-resolution of $M$, so $n=\bar{\lambda}(M) \geq s+k+1$. Therefore, $\bar{\lambda}\left(L_{k}\right)=s \leq n-k-1$. On the other hand $s \geq n-k-1$, so we see that equality holds and $\bar{\lambda}\left(L_{k}\right)=n-k-1$.

In the case $\bar{\lambda}(M)=\infty$ we have the following corollary. 
Corollary 2.3. If the cotorsion theory $(\mathcal{F}, \mathcal{C})$ satisfies $H C$ and $\bar{\lambda}(M)=\infty$ then there is an infinite special $\mathcal{F}$-resolution $\ldots \longrightarrow F_{n} \stackrel{d_{n}}{\longrightarrow} F_{n-1} \longrightarrow \ldots \longrightarrow F_{1} \stackrel{d_{1}}{\longrightarrow} F_{0} \stackrel{d_{0}}{\longrightarrow} M \longrightarrow 0$ of $M$.

Proof. Since $\bar{\lambda}(M) \geq 0$, there is a special $\mathcal{F}$-precover $F_{0} \stackrel{f_{0}}{\longrightarrow} M \longrightarrow 0$ of $M$. Since $\bar{\lambda}(M) \geq$ $1, \bar{\lambda}\left(\operatorname{Ker} f_{0}\right) \geq 0$ by Theorem 2.2 , so there is a special $\mathcal{F}$-precover $F_{1} \stackrel{f_{1}}{\longrightarrow} \operatorname{Ker} f_{0} \longrightarrow 0$ of $\operatorname{Ker} f_{0}$. Now $\bar{\lambda}(M) \geq 2$, therefore $\bar{\lambda}\left(\operatorname{Ker} f_{0}\right) \geq 0$ and Ker $f_{1}$ has a special $\mathcal{F}$-precover. Continuing in this way an infinite special $\mathcal{F}$-resolution can be constructed for $M$.

We would like to give the following result (which follows immediately from Theorem 8.6.16 in [5]) in connection with Theorem 3.8 of [1]. Recall that a cotorsion theory $(\mathcal{F}, \mathcal{C})$ is said to satisfy the extended hereditary condition $(E H C)$ if it satisfies $H C, \operatorname{gl} . \operatorname{dim} R<\infty$ and every module from $\mathcal{C}$ has a special $\mathcal{F}$-precover (or equivalently, every module from $\mathcal{F}$ has a special $\mathcal{C}$-preenvelope) (see [1]). Here the given condition that $E H C$ should hold can now be replaced by the condition that $\bar{\lambda}\left(M^{\prime \prime}\right) \geq 1$.

Corollary 2.4. If the cotorsion theory $(\mathcal{F}, \mathcal{C})$ satisfies $H C$ and in the short exact sequence $0 \rightarrow M^{\prime} \rightarrow M \rightarrow M^{\prime \prime} \rightarrow 0, M$ has a special $\mathcal{F}$-precover and $\bar{\lambda}\left(M^{\prime \prime}\right) \geq 1$, then $M^{\prime}$ has a special $\mathcal{F}$-precover.

Now we study the case when every module from $\mathcal{C}$ has a special $\mathcal{F}$-precover.

Lemma 2.5. If every module from $\mathcal{C}$ has a special $\mathcal{F}$-precover, then for every module $M$ either $\bar{\lambda}(M)=-1$ or $\bar{\lambda}(M)=\infty$. In particular, $\bar{\lambda}(C)=\infty$ for every $C$ from $\mathcal{C}$.

Proof. If $\bar{\lambda}(M) \neq-1$, i. e. $M$ has a special $\mathcal{F}$-precover $0 \rightarrow C_{0} \rightarrow F_{0} \rightarrow M \rightarrow 0$, then $C_{0}$ has a special $\mathcal{F}$-precover $0 \rightarrow C_{1} \rightarrow F_{1} \rightarrow C_{0} \rightarrow 0$ and so on, $C_{n}$ has a special $\mathcal{F}$-precover $0 \rightarrow C_{n+1} \rightarrow F_{n+1} \rightarrow C_{n} \rightarrow 0$. Yoneda product of these short exact sequences gives an infinite special $\mathcal{F}$-resolution

$$
\ldots \rightarrow F_{n} \rightarrow F_{n-1} \rightarrow \ldots \rightarrow F_{1} \rightarrow F_{0} \rightarrow M \rightarrow 0
$$

of $M$. So $\bar{\lambda}(C)=\infty$. The second statement is obvious.

Proposition 2.6. If the cotorsion theory $(\mathcal{F}, \mathcal{C})$ satisfies $H C$ and every module from $\mathcal{C}$ has a special $\mathcal{F}$-precover, then for every module $M$ with finite injective dimension, $\bar{\lambda}(M)=\infty$.

Proof. Let inj. $\operatorname{dim} M=n$ and

$$
0 \longrightarrow M \longrightarrow I_{0} \longrightarrow \ldots \longrightarrow I_{n-1} \longrightarrow I_{n} \longrightarrow 0
$$

be an injective resolution of $M$. This sequence can be represented as an Yoneda product of short exact sequences

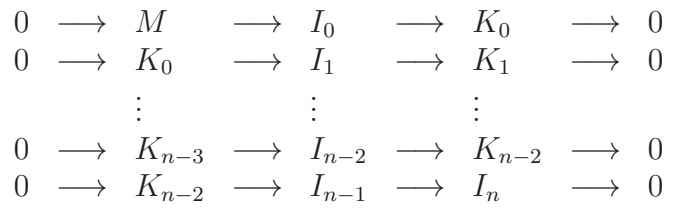


Since $\bar{\lambda}\left(I_{n-1}\right)=\bar{\lambda}\left(I_{n}\right)=\infty$, applying Corollary 2.4 to the last row we obtain that $K_{n-2}$ has a special $\mathcal{F}$-precover and by Lemma $2.5 \bar{\lambda}\left(K_{n-2}\right)=\infty$. Similarly Corollary 2.4 and Lemma 2.5 gives $\bar{\lambda}\left(K_{n-3}\right)=\infty$. Continuing in this way we see that $\bar{\lambda}(M)=\infty$.

The dual results hold for $\bar{\mu}$-dimensions.

The following corollary of 2.6 gives an improvement of Proposition 3.7 in [1].

Corollary 2.7. If the cotorsion theory $(\mathcal{F}, \mathcal{C})$ satisfies EHC, then $\bar{\lambda}(M)=\bar{\mu}(M)=\infty$ for every module $M$.

Lemma 2.8. If the cotorsion theory $(\mathcal{F}, \mathcal{C})$ satisfies $\mathrm{HC}$, then $\operatorname{Ext}^{n}(F, C)=0$ for every $F \in \mathcal{F}, C \in \mathcal{C}$ and $n \geq 1$.

Proof. Let $C \in \mathcal{C}$. By induction on $n$ we prove that $\operatorname{Ext}^{n}(F, C)=0$ for every $F \in \mathcal{F}$. For $n=1,2$ it satisfies by the definitions. Let $n \geq 3$ and suppose that the equality satisfies for every $k<n$ and let $F \in \mathcal{F}$. Take any short exact sequence $0 \rightarrow A \rightarrow P \rightarrow F \rightarrow 0$ with projective $P$. Then $A \in \mathcal{F}$ since $(\mathcal{F}, \mathcal{C})$ satisfies $H C$. Therefore from the exact sequence

$$
\ldots \longrightarrow \operatorname{Ext}^{n-1}(A, C) \longrightarrow \operatorname{Ext}^{n}(F, C) \longrightarrow \operatorname{Ext}^{n}(P, C) \longrightarrow \ldots
$$

we conclude that $\operatorname{Ext}^{n}(F, C)=0$.

Theorem 2.9. Suppose that the cotorsion theory $(\mathcal{F}, \mathcal{C})$ satisfies $\mathrm{HC}$ and $\mathrm{gl}$. $\operatorname{dim} R=n<$ $\infty$. If $\lambda(M) \geq n-1(\bar{\lambda}(M) \geq n-1)$, then $\lambda(M)=\infty \bar{\lambda}(M)=\infty)$.

Proof. Suppose that $\lambda(M) \geq n-1$, i.e. we have a partial $\mathcal{F}$-resolution $F_{n-1} \stackrel{d_{n-1}}{\longrightarrow} F_{n-2} \longrightarrow$ $\ldots \longrightarrow F_{1} \stackrel{d_{1}}{\longrightarrow} F_{0} \stackrel{d_{0}}{\longrightarrow} M \longrightarrow 0$ with each $F_{i} \in \mathcal{F}$. Then we have the following short exact sequences:

$$
\begin{aligned}
& 0 \longrightarrow K_{n-1} \longrightarrow F_{n-1} \longrightarrow K_{n-2} \longrightarrow 0 \\
& 0 \longrightarrow K_{n-2} \longrightarrow F_{n-2} \longrightarrow K_{n-3} \longrightarrow 0 \\
& \vdots \\
&
\end{aligned}
$$

where $K_{i}=\operatorname{Ker} d_{i}$ for $i=0,1,2, \ldots, n-1$. For every $C \in \mathcal{C}$ we have the following exact sequences:

$$
\begin{aligned}
& \operatorname{Ext}^{1}\left(F_{n-1}, C\right) \longrightarrow \operatorname{Ext}^{1}\left(K_{n-1}, C\right) \longrightarrow \operatorname{Ext}^{2}\left(K_{n-2}, C\right) \longrightarrow \operatorname{Ext}^{2}\left(F_{n-1}, C\right) \\
& \operatorname{Ext}^{2}\left(F_{n-2}, C\right) \longrightarrow \operatorname{Ext}^{2}\left(K_{n-2}, C\right) \longrightarrow \operatorname{Ext}^{3}\left(K_{n-3}, C\right) \longrightarrow F^{3}\left(F_{n-2}, C\right) \\
& \vdots \\
& \operatorname{Ext}^{n-1}\left(F_{1}, C\right) \longrightarrow \operatorname{Ext}^{n-1}\left(K_{1}, C\right) \longrightarrow \operatorname{Ext}^{n}\left(K_{0}, C\right) \\
& \operatorname{Ext}^{n}\left(F_{0}, C\right) \longrightarrow \operatorname{Ext}^{n}\left(K_{0}, C\right) \longrightarrow \operatorname{Ext}^{n}\left(F_{1}, C\right)
\end{aligned}
$$

Since $\operatorname{Ext}^{1}\left(F_{n-1}, C\right)=\operatorname{Ext}^{2}\left(F_{n-1}, C\right)=\operatorname{Ext}^{2}\left(F_{n-2}, C\right)=\operatorname{Ext}^{3}\left(F_{n-2}, C\right)=\ldots=\operatorname{Ext}^{n}\left(F_{0}, C\right)=$ $\operatorname{Ext}^{n+1}\left(F_{0}, C\right)=0$ we have the isomorphisms

$$
\begin{gathered}
\operatorname{Ext}^{1}\left(K_{n-1}, C\right) \cong \operatorname{Ext}^{2}\left(K_{n-2}, C\right) \cong \operatorname{Ext}^{3}\left(K_{n-3}, C\right) \cong \ldots \cong \operatorname{Ext}^{n-1}\left(K_{1}, C\right) \cong \\
\cong \operatorname{Ext}^{n}\left(K_{0}, C\right) \cong \operatorname{Ext}^{n+1}(M, C) .
\end{gathered}
$$


But gl. $\operatorname{dim} R=n$, so $\operatorname{Ext}^{1}\left(K_{n-1}, C\right) \cong \operatorname{Ext}^{n+1}(M, C) \cong 0$. Therefore $K_{n-1} \in \mathcal{F}$. So we have an infinite $\mathcal{F}$-resolution of $M$ :

$$
\ldots \rightarrow 0 \longrightarrow K_{n-1} \longrightarrow F_{n-1} \longrightarrow \ldots \longrightarrow F_{1} \stackrel{d_{1}}{\longrightarrow} F_{0} \stackrel{d_{0}}{\longrightarrow} M \longrightarrow 0
$$

The proof of the equality $\bar{\lambda}(M)=\infty$ is similar.

The dual results hold for for the $\mu-$ and $\bar{\mu}-$ dimensions of modules.

\section{Relations Between $\bar{\lambda}$ - And $\lambda$-Dimensions, And $\bar{\mu}$ - And $\mu$-Dimensions}

In this section we aim to give inequalities between the $\bar{\lambda}$ - and $\lambda$-dimensions of modules in a short exact sequence. These inequalities are similar to the inequalities involving only the $\lambda$-dimensions, or the $\bar{\lambda}$-dimensions in [6]. We use Theorem 3.1 of [1] to prove the following theorem which is similar to Theorem 8.6.9 of [5]. In our case the complex is not necessarily $\operatorname{Hom}(\mathcal{F},-)$ exact, but the given cotorsion theory satisfies $H C$.

Theorem 3.1. If the cotorsion theory $(\mathcal{F}, \mathcal{C})$ satisfies $H C$ and $0 \rightarrow M^{\prime} \rightarrow M \rightarrow M^{\prime \prime} \rightarrow 0$ is exact then:

(1) $\lambda(M) \geq \min \left(\bar{\lambda}\left(M^{\prime}\right), \lambda\left(M^{\prime \prime}\right)\right)$,

(2) $\lambda\left(M^{\prime}\right) \geq \min \left(\lambda(M), \bar{\lambda}\left(M^{\prime \prime}\right)-1\right)$.

Proof. (1) We modify the proof of Theorem 2.1 as follows. Let $\min \left(\bar{\lambda}\left(M^{\prime}\right), \lambda\left(M^{\prime \prime}\right)\right)=n$. By induction on $n$ we prove that $\lambda(M) \geq n$. Again for $n=-1$ there is nothing to prove. Assume that for $n \leq k$ the inequality holds and let $n=k+1$. There is a special $\mathcal{F}$-resolution $F_{k}^{\prime} \rightarrow F_{k-1}^{\prime} \rightarrow \ldots \rightarrow F_{1}^{\prime} \rightarrow F_{0}^{\prime} \rightarrow M^{\prime} \rightarrow 0$ for $M^{\prime}$ and an $\mathcal{F}$-resolution $F_{k}^{\prime \prime} \rightarrow F_{k-1}^{\prime \prime} \rightarrow \ldots \rightarrow F_{1}^{\prime \prime} \rightarrow F_{0}^{\prime \prime} \rightarrow M^{\prime \prime} \rightarrow 0$ for $M^{\prime \prime}$. For every $F \in \mathcal{F}$ applying $\operatorname{Hom}(F,-)$ to the commutative exact diagram:

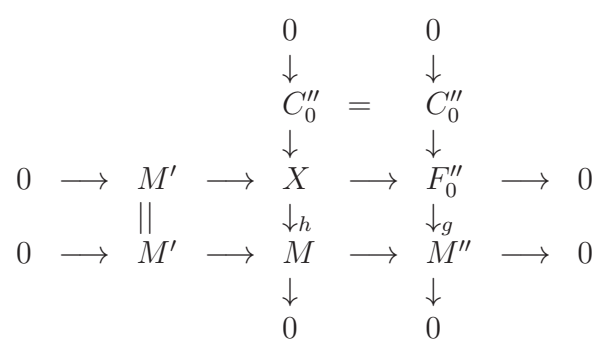

we have the following diagram with exact rows:

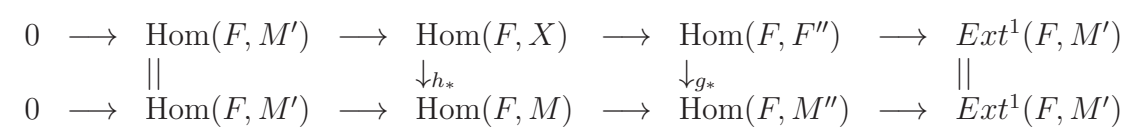

Since $g_{*}$ is epic, $h_{*}$ is also epic by the Five Lemma. Furthermore since Ker $e \cong \operatorname{Ker} f=$ $C_{0}^{\prime} \in \mathcal{C}$, applying $\operatorname{Hom}(F,-)$ to the exact sequence $0 \rightarrow C_{0}^{\prime} \longrightarrow F_{0} \stackrel{e}{\longrightarrow} X \longrightarrow 0$ we obtain the following exact sequence 


$$
\cdots \longrightarrow \operatorname{Hom}\left(F, F_{0}\right) \stackrel{e_{*}}{\longrightarrow} \operatorname{Hom}(F, X) \longrightarrow \operatorname{Ext}^{1}\left(F, C_{0}^{\prime}\right)=0
$$

from which we conclude that $e_{*}$ is also epic. Then $(h \circ e)_{*}=h_{*} \circ e_{*}$ is epic and therefore $0 \longrightarrow C_{0} \longrightarrow F_{0} \stackrel{\text { hoe }}{\longrightarrow} M \longrightarrow 0$ is $\operatorname{Hom}(\mathcal{F},-)$ exact.

Here $F_{0}^{\prime}$ and $F_{0}^{\prime \prime}$ in $\mathcal{F}$ gives us that $F_{0} \in \mathcal{F}$. Now if $k=0$ then $F_{0} \stackrel{h \circ e}{\longrightarrow} M$ is an $\mathcal{F}$ precover of $M$, so $\lambda(M) \geq 0$. If $k \geq 1$, then $\min \left(\bar{\lambda}\left(C_{0}^{\prime}\right), \lambda\left(C_{0}^{\prime \prime}\right)\right) \geq k$, and so by the inductive assumption $\lambda\left(C_{0}\right) \geq k$, that is, $C_{0}$ has a $\mathcal{F}$-resolution $F_{k} \longrightarrow F_{k-1} \longrightarrow \ldots \longrightarrow$ $F_{1} \stackrel{u}{\longrightarrow} C_{0} \longrightarrow 0$, therefore $F_{k} \longrightarrow F_{k-1} \longrightarrow \ldots \longrightarrow F_{1} \stackrel{v \circ u}{\longrightarrow} F_{0} \longrightarrow M \longrightarrow 0$ forms an $\mathcal{F}$-resolution of $M$. That is, $\lambda(M) \geq k+1$.

(2) Let $\min \left(\lambda(M), \bar{\lambda}\left(M^{\prime \prime}\right)-1\right)=n$. Then there is an exact sequence, $0 \rightarrow C_{0}^{\prime \prime} \rightarrow$ $F_{0}^{\prime \prime} \rightarrow M_{0}^{\prime \prime} \rightarrow 0$ with $F_{0}^{\prime \prime} \in \mathcal{F}$ and $C_{0}^{\prime \prime} \in \mathcal{C}$ and $\bar{\lambda}\left(C_{0}^{\prime \prime}\right) \geq n$. We have an exact commutative diagram:

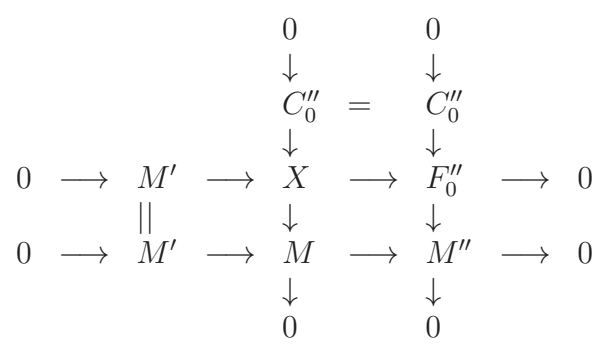

By 1) we have that $\lambda(X) \geq n$. Then we have a $\operatorname{Hom}(\mathcal{F},-)$ exact sequence $0 \rightarrow A_{0} \rightarrow$ $F_{0} \rightarrow X \rightarrow 0$ with $F_{0} \in \mathcal{F}$ and $\lambda\left(A_{0}\right) \geq n-1$. From this we get the following exact commutative diagram;

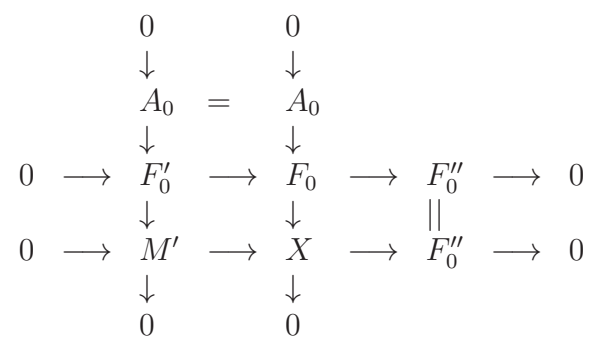

One can easily verify by means of the Five Lemma (using the techniques of the proof of the first part), that the sequence $0 \rightarrow A_{0} \rightarrow F_{0}^{\prime} \rightarrow M^{\prime} \rightarrow 0$ is $\operatorname{Hom}(\mathcal{F},-)$ exact. $F_{0}$, $F_{0}^{\prime \prime} \in \mathcal{F}$ means that because of $H C, F_{0}^{\prime} \in \mathcal{F}$. Now if $F_{n-1}^{\prime} \rightarrow \ldots \rightarrow F_{1}^{\prime} \rightarrow A_{0} \rightarrow 0$ is a left $\mathcal{F}$-resolution of $A_{0}$, then $F_{n-1}^{\prime} \rightarrow \ldots \rightarrow F_{1}^{\prime} \rightarrow F_{0} \rightarrow M^{\prime} \rightarrow 0$ is a left $\mathcal{F}$-resolution of $M^{\prime}$. Therefore $\lambda\left(M^{\prime}\right) \geq n$. 
The dual results for $\bar{\mu}$ - and $\mu$-dimensions derived in a similar way and we state the theorem without proof.

Theorem 3.2. If the cotorsion theory $(\mathcal{F}, \mathcal{C})$ satisfies $H C$ and $0 \rightarrow M^{\prime} \rightarrow M \rightarrow M^{\prime \prime} \rightarrow 0$ is exact then;

1) $\mu(M) \geq \min \left(\mu\left(M^{\prime}\right), \bar{\mu}\left(M^{\prime \prime}\right)\right)$,

2) $\mu\left(M^{\prime \prime}\right) \geq \min \left(\bar{\mu}\left(M^{\prime}\right)-1, \mu(M)\right)$.

\section{REFERENCES}

[1] K. D. Akıncı and R. Alizade, Special precovers in cotorsion theories, Proc. Edin. Math. Soc. 45:2 (2002), 411-420.

[2] Cotorsion theories and resolutions, International Congress of Math., Abstracts of Short Communications and Poster Sessions (2002), 9.

[3] R. Alizade, Inheritance of the properties of coprojectivity and coinjectivity for certain proper classes,(Russian), Izv. Akad. Nauk Azerbaijan. SSR Ser. Fiz.-Tekhn. Mat. Nauk 4:5 (1983), 3-7.

[4] S. Bazzoni and L. Salce, On strongly flat modules over integral domains, Rocky Mountan J. Math. 34:2 (2004), 417-439.

[5] E. Enochs and O. M. G. Jenda, Relative homological algebra, Walter de Gruyter-New York, 2000.

[6] E.Enochs, O. M. G. Jenda, and L. Oyonarte, $\lambda$ and $\mu$-Dimensions of Modules, Rend. Sem. Mat. Univ. Padova 105 (2001), 111-123.

[7] E.Matlis, Torsion-Free Modules, Chicago Lectures in Mathematics, University of Chicago Press, Chicago-London, 1972.

[8] L.Salce, Cotorsion theories for abelian groups, Symp. Math. 23 (1972), 12-32.

[9] J.Xu, Flat covers of modules, Lecture Notes in Mathematics, 1634, Springer Verlag, 1996.

E-MAIL:KARENAKINCI QYAHOO. CO. UK

Izmir Institute of Technology,, Department of Mathematics,, GülbahÇeköyü, 35437 , Urla,, IZmir, TURKEY, E-Mail: Rafailalizade@iyte.edu.tr 